\title{
Tipping the Extracellular Matrix Balance during Heart Failure Progression: Do We Always Go Right?
}

\author{
Ying Ann Chiao a, b Yu-Fang Jin ${ }^{c}$ Merry L. Lindsey ${ }^{a}$ \\ a Division of Cardiology, Department of Medicine, and ${ }^{b}$ Department of Biochemistry, University of Texas \\ Health Science Center at San Antonio, and ' Department of Electrical Engineering, University of Texas at \\ San Antonio, San Antonio, Tex., USA
}

Matrix metalloproteinases (MMPs) are highly implicated regulators of cardiac remodeling, and multiple teams have measured MMP and tissue inhibitor of metalloproteinase (TIMP) levels in various cardiac disease models. Animal and human studies have demonstrated that MMPs increase and are positively associated with remodeling parameters [1-3]. Mice with targeted deletion of specific MMPs or the TIMPs have also incriminated particular MMPs and TIMPs, including MMP-2 and 9 and TIMP-1, in adverse remodeling following myocardial infarction [4-7]. However, MMP inhibition strategies have not proven effective in limiting post-myocardial infarction remodeling [8]. At this juncture, a crucial question to ask is: if MMPs and extracellular matrix (ECM) degradation are on the right side of the scale and TIMPs and ECM accumulation are on the left side, is interfering with the right side (using MMP inhibitors) the only viable therapeutic strategy to tip the balance?

In this issue of Cardiology, Yang et al. [9] examine levels of MMP-2, MMP-3, and MMP-9, as well as TIMP-1 and fibronectin, in patients with congestive heart failure. Compared to control levels, plasma and ventricular levels of MMP-2, 3, and 9 progressively increased with severity of heart failure, while levels of TIMP-1 and full-length fibronectin declined. The change in MMP and TIMP expression levels correlated strongly with the decline in NYHA class. While these results place these analytes at the scene of the crime, several questions remain to be answered before a guilty plea can be entered.

For one, there is a need to measure other MMPs and TIMPs that will provide a more complete picture of the ECM turnover status in the failing left ventricle. While the authors state that 'only the most important myocardial MMPs ... were evaluated', the conclusion that MMP2,3 , and 9 are most important is derived from the fact that they are the most frequently measured. Whether frequency equals importance has not been established for the MMP family. In particular, information on additional inflammatory-related MMPs $(7,8,12,14,16,28)$ would fill in crucial details for the detectives.

While the authors demonstrate a decrease in TIMP-1 levels in this study, plasma TIMP-1 increases during different stages of hypertension and myocardial infarction $[10,11]$. In fact, increased TIMP-1 has been proposed as

\section{KARGER}

(ㄷ) 2010 S. Karger AG, Basel

Fax +41613061234 E-Mail karger@karger.ch www.karger.com www.karger.com/crd
Merry L. Lindsey, PhD

Cardiology Division, Department of Medicine

University of Texas Health Science Center at San Antonio

7703 Floyd Curl Drive, Mail Code 7872, San Antonio, TX 78229-3900 (USA)

Tel. +1 210567 4673, Fax +1 210567 6960, E-Mail lindseym@uthscsa.edu 
a cardiac biomarker of remodeling $[12,13]$. Therefore, it is surprising that TIMP-1 levels are gradually reduced over the course of heart failure. The decrease in TIMP-1 levels suggests an increased ECM degradative capacity with more severe heart failure. It is interesting that the coefficient of variation for the MMPs decreases with increasing NYHA class (indicating a less variable response), while the coefficient of variation for TIMP-1 increases with heart failure progression (indicating a more variable response). This result could mean that TIMP-1 values may separate out patients even within a particular heart failure class. This observation also highlights the fact that we still do not have a firm idea what role TIMPs play during cardiac remodeling. We know that TIMPs inhibit MMPs, but TIMPs play non-MMP-mediated roles as well. TIMPs can signal through cell surface receptors in an MMP-independent manner [1], with one effect being increased cell proliferation [14]. Therefore, more attention should be given to exactly what the TIMP family members are doing in the heart failure setting to determine if their alibis as protective components hold up.

MMP to TIMP ratios are often presented in publications as evidence for or against a tip in the balance of MMPs to TIMPs. In this article, all ratios steadily increased with heart failure class, suggesting an increase in MMP degradative capacity. The issue with this interpretation is that there are 25 MMPs and 4 TIMPs identified to date, such that randomly selecting 1 MMP and 1 TIMP and determining the ratio between the 2 is arbitrary and may not reflect the entire composition of the plasma or tissue. If the other TIMPs increase to a greater extent compared to the decrease in TIMP-1 (or the converse for any of the MMPs), then the balance is actually the opposite of what is presented. The interpretation of ratio information, therefore, should be taken with caution. The jury is still out on whether total MMP levels, MMP bound to TIMP levels, or MMP to TIMP ratios prove the most informative way to present MMP and TIMP results.

The Yang laboratory reported that full-length fibronectin levels fell as NYHA class increased. The relevance of decreased fibronectin in the ventricular samples needs to be more fully examined. Was the decrease in fulllength levels accompanied by an increase in the generation of fibronectin fragments? This would be consistent with an increase in MMP-2 and MMP-9 levels, as fibronectin is a substrate of both MMPs [15]. The Knowlton laboratory has shown that fibronectin fragments can enhance survival of injured cardiac myocytes [16], while fibronectin fragments have also been shown to induce the release of cytokines and MMPs from epithelial cells [17]. Likewise, increased collagen deposition and the increased generation of collagen fragments have both been linked to adverse events, indicating that multiple ECMs can be proteolytically processed to generate peptides with potent biological activity. Decreases in full-length fibronectin versus increases in 120 - or $40-\mathrm{kDa}$ matricryptin fragments of fibronectin may signify enhanced downstream signaling events. However, few studies have addressed cardiac matricryptin generation, and this field is ripe for investigation. Specifically, whether matricryptin generation serves as a link to connect the two sides of the balance and whether fine tuning this connection would provide a therapeutically viable option remains to be evaluated.

A strength of this study is that genders were equally represented, because heart failure occurs in both genders about equally. In addition, the heart failure patients were separated by NYHA class. While changes are progressive among the classes, whether direct cause and effect relationships between the change in MMP, TIMP, and fibronectin levels caused the decline in LV function (and how this was accomplished) remains to be assessed. Animal models using time courses and variations in disease severity to modulate levels will help to sort this out.

In addition, a limitation that needs to be taken into consideration is the fact that only one time point was evaluated. While it could be argued that class is an indirect reflection of time, longitudinally following the same individual will allow us to determine if levels are always high/low or whether levels change over time. A static measurement of a chronic process only tells us where the process is at that snap shot in time, not before and after the time under examination. One caveat for this study is the fact that the mean ages of the 4 groups ranged from 42 to 48 years old, indicating that this study was conducted on a relatively young cohort. Additional studies evaluating older patients will provide information on the effects of aging superimposed on heart failure progression.

In summary, the study by Yang et al. extends the results of several previous publications to suggest several possible mechanisms whereby MMPs and TIMPs can be regulated over the course of heart failure progression. While MMP and TIMP levels are often presented in snap shot results, both are likely in states of flux over the course of disease. Future studies will need to delineate the full complement of mechanisms that regulate LV function and the downstream consequences of altered MMP and TIMP functions, in order to determine if in- 
hibiting or stimulating ECM remodeling at particular phases would result in a positive outcome in the setting of heart failure. Included in this list are the role of fibronectin in remodeling and the complete understanding of TIMP functions. Tipping the left side, by increasing TIMP levels, needs to be given consideration as a therapeutic option.

\section{Acknowledgements}

The authors acknowledge grant support from the UT Health Science Center, San Antonio Translational Science Training Program (to Y.A.C.); NIH SC2 HL101430 (to Y.-F.J.); and NIH R01 HL75360, AHA Grant-in-Aid 0855119F, the Veterans Administration, Novartis, and the Max and Minnie Tomerlin Voelcker Fund (to M.L.L.).

\section{References}

1 Vanhoutte D, Heymans S: TIMPs and cardiac remodeling: 'embracing the MMP-independent-side of the family'. J Mol Cell Cardiol 2010;48:445-453.

-2 Zamilpa R, Lindsey ML: Extracellular matrix turnover and signaling during cardiac remodeling following MI: causes and consequences. J Mol Cell Cardiol 2010;48:558563.

-3 Spinale FG: Myocardial matrix remodeling and the matrix metalloproteinases: influence on cardiac form and function. Physiol Rev 2007;87:1285-1342.

-4 Hayashidani S, Tsutsui H, Ikeuchi M, Shiomi T, Matsusaka H, Kubota T, Imanaka-Yoshida $\mathrm{K}$, Itoh T, Takeshita A: Targeted deletion of MMP-2 attenuates early LV rupture and late remodeling after experimental myocardial infarction. Am J Physiol 2003;285:H1229H1235.

5 Lindsey ML, Escobar GP, Dobrucki LW, Goshorn DK, Bouges S, Mingoia JT, McClister DM Jr, Su H, Gannon J, MacGillivray C, Lee RT, Sinusas AJ, Spinale FG: Matrix metalloproteinase-9 gene deletion facilitates angiogenesis after myocardial infarction. Am J Physiol 2006;290:H232-H239.

6 Creemers EEJM, Davis JN, Parkhurst AM, Leenders P, Dowdy KB, Hapke E, Hauet AM, Escobar PG, Cleutjens JPM, Smits JFM, Daemen MJAP, Zile MR, Spinale FG: Deficiency of TIMP-1 exacerbates LV remodeling after myocardial infarction in mice. Am J Physiol 2003;284:H364-H371.
7 Matsumura S, Iwanaga S, Mochizuki S, Okamoto H, Ogawa S, Okada Y: Targeted deletion or pharmacological inhibition of MMP2 prevents cardiac rupture after myocardial infarction in mice. J Clin Invest 2005;115: 599-609.

8 Peterson JT: The importance of estimating the therapeutic index in the development of matrix metalloproteinase inhibitors. Cardiovasc Res 2006;69:677-687.

-9 Yang D, Ma S, Tan Y, Chen Y, Li D, Tang B, Chen J, Su X, Li G, Zhang X, Yang Y: Imbalance of matrix metalloproteinases/tissue inhibitor of metalloproteinase-1 and loss of fibronectin expression in patients with congestive heart failure. Cardiology 2010;116: 133-141.

10 Lindsay MM, Maxwell P, Dunn FG: TIMP-1: a marker of left ventricular diastolic dysfunction and fibrosis in hypertension. Hypertension 2002;40:136-141.

11 Inokubo $\mathrm{Y}$, Hanada $\mathrm{H}$, Ishizaka $\mathrm{H}$, Fukushi T, Kamada T, Okumura K: Plasma levels of matrix metalloproteinase- 9 and tissue inhibitor of metalloproteinase- 1 are increased in the coronary circulation in patients with acute coronary syndrome. Am Heart J 2001; 141:211-217.

12 Cavusoglu E, Ruwende C, Chopra V, Yanamadala S, Eng C, Clark LT, Pinsky DJ, Marmur JD: Tissue inhibitor of metalloproteinase-1 (TIMP-1) is an independent predictor of all-cause mortality, cardiac mortality, and myocardial infarction. Am Heart J 2006; 151: 1101.e1-1101.e8.
13 Frantz S, Stork S, Michels K, Eigenthaler M, Ertl G, Bauersachs J, Angermann CE: Tissue inhibitor of metalloproteinases levels in patients with chronic heart failure: an independent predictor of mortality. Eur J Heart Fail 2008;10:388-395.

14 Lovelock JD, Baker AH, Gao F, Dong JF, Bergeron AL, McPheat W, Sivasubramanian N, Mann DL: Heterogeneous effects of tissue inhibitors of matrix metalloproteinases on cardiac fibroblasts. Am J Physiol 2005; 288:H461-H468.

15 Morodomi T, Ogata Y, Sasaguri Y, Morimatsu M, Nagase H: Purification and characterization of matrix metalloproteinase 9 from U937 monocytic leukaemia and HT1080 fibrosarcoma cells. Biochem J 1992;285:603611.

16 Trial J, Rossen RD, Rubio J, Knowlton AA: Inflammation and ischemia: macrophages activated by fibronectin fragments enhance the survival of injured cardiac myocytes. Exp Biol Med 2004;229:538-545.

17 Austin BA, Liu B, Li Z, Nussenblatt RB: Biologically active fibronectin fragments stimulate release of MCP-1 and catabolic cytokines from murine retinal pigment epithelium. Invest Ophthalmol Vis Sci 2009;50:2896-2902. 